\title{
A low-noise and scalable FPGA- based analog signal generator for quantum gas experiments
}

\section{Conference Paper}

\section{Author(s):}

Pahl, David; Pahl, Lukas; Mustafa, Enis; Liu, Zhenning; Fabritius, Philipp; Clements, Peter; Akin, Abdulkadir; Mohan, Jeffrey (iD; Esslinger, Tilman

Publication date:

2021

\section{Permanent link:}

https://doi.org/10.3929/ethz-b-000503065

\section{Rights / license:}

In Copyright - Non-Commercial Use Permitted

\section{Originally published in:}

https://doi.org/10.1109/QCE52317.2021.00073

\section{Funding acknowledgement:}

182650 - Interplay between Topology, Interactions and Dissipation in Driven Quantum Many-Body Systems (SNF) 742579 - Mass, heat and spin transport in interlinked quantum gases (EC) 


\title{
A low-noise and scalable FPGA-based analog signal generator for quantum gas experiments.
}

\author{
D. Pahl', L. Pahl*, E. Mustafa*, Z. Liu ${ }^{\dagger}$, P. Fabritius ${ }^{\dagger}$, J. Mohan ${ }^{\dagger}$, P. Clements ${ }^{\dagger}$, A. Akin ${ }^{\dagger}$, T. Esslinger ${ }^{\dagger}$ \\ ${ }^{*}$ Department of Information Technology and Electrical Engineering, ETH Zürich, 8092 Zürich, Switzerland \\ ${ }^{\dagger}$ Department of Physics, ETH Zürich, 8093 Zürich, Switzerland
}

\begin{abstract}
Achieving high fidelity for the measurement and control of quantum experiments imposes strict requirements on the precision and stability of surrounding electronics. Controlling electronics from a central device is more challenging when they are distributed in a laboratory and require analog signals where effects like ground loops and radiative cross-talk can limit their performance. Here, we present our design to address these challenges with a flexible and scalable analog signal generator. Our design is based on a field-programmable gate array (FPGA) development board, a custom PCB hosting a digital-to-analog converter (DAC) with 20 bit precision at 1 MSPS, and a custom breakout board. The FPGA development board accepts data from a master PC via TCP/IP where a user programs the waveform and sampling rate of each output channel and writes the data to on-board RAM. At runtime, the direct memory access (DMA) and Serial Peripheral Interface (SPI) modules inside the FPGA stream data to the custom DAC board via an Ethernet cable carrying the samples as differential signals along with the supply voltage. We designed the DAC board to be resistant to digital and analog noise by separating ground planes to prevent ground loops and by using high-precision and low-noise power supplies and voltage reference circuits. External trigger and clock inputs can be used to synchronize the DACs and multiple FPGAs. The time resolution and precision of our solution is optimized for experiments on quantum gases though it is flexible and can be adapted for many more applications.
\end{abstract}

\section{INTRODUCTION}

State of the art quantum gas experiments [1], [2] rely on the precise control of experimental parameters like magnetic fields, laser frequencies, and laser powers to control and probe quantum systems. These parameters, typically set by an analog control signal and PID controller, need to be changed to the required conditions over several orders of magnitude at speeds ranging from seconds to microseconds depending on the dynamics of the system. Furthermore, these signals often must be extremely stable as noise introduced by e.g. controlling electronics or cross-talk can reduce the lifetime of such systems [3]. Moreover, the complexity of the experiments requires the control of more than 50 devices synchronously which requires a scalable solution with a central controller.

The most common way of controlling quantum gas experiments is based on one or more central units with multiple digital and analog outputs connected to the experiment via coaxial cables carrying analog signals ([4], [5], [6]). Our approach to address the aforementioned challenges with this architecture is inspired by the control system developed in [7] where we improve on the precision and output range on the DAC side and simplify the real-time controller by using a commercially available FPGA development board [8]. The full system will feature multiple FPGAs synchronized to a central clock, combined with breakout boards for the connection to up to a total of 10 DAC boards - each with a separate, isolated ground to remove ground loops. The implementation of a dynamical sampling rate and individually updated samples for each channel at up to 1 MSPS reduces the needed storage space and data transfer bandwidth from the central control PC. The proposed system including our custom FPGA design, break-out board and DAC board is presented.

\section{SYSTEM OVERVIEW}

The high-level architecture of our signal generator and how it is integrated in the experiment is shown in Figure 1. The user programs the experimental sequence on the PC by defining the behavior of each device (digital/analog outputs, frequency sources, power supplies, etc.) at each point in time including the waveforms of each channel of the signal generator. The PC then programs each device via PCI, PCIe, Ethernet, USB, etc. and initiates the execution synchronously. This is currently done with a home-built experiment control software [9], but one could use any program capable of TCP/IP communication. The waveforms are written to on-board RAM by the programmable software of the FPGA and, at runtime, the programmable logic streams the samples as SPI signals along with a supply voltage via Ethernet cables to the DAC boards which are directly connected to the devices they drive e.g. PID controller. Transmitting the signals digitally rather than analogically has two distinct advantages: (1) the low voltage differential signal (LVDS) carried by the shielded, twisted pairs in the Ethernet cable are virtually impervious to radiation noise present in the laboratory which would corrupt analog signals in long coaxial cables and (2) the grounds of the PID controller and FPGA board can be separated to remove ground loops which could introduce significant noise in delicate signals, principally mains hum.

The waveform of the signal generator is defined not only by the output voltage at each time step but also the sampling rate. Because quantum gas experiments contain many steps with vastly different timescales - for example, loading a magnetooptical trap can take several seconds while the physics of interest at the end of the experiment can finish in just a few microseconds - we have implemented the feature that the user can change the sampling rate for each channel separately at any point in the waveform. Example waveforms 
with this dynamical sampling rate are shown in Figure 2. Each waveform is characterized by metadata, which is used by the FPGA to control the DACs at execution time as is explained in Section III.

We have designed the generator to operate up to $1 \mathrm{MSPS}$. This requires a minimum SPI clock rate of $25 \mathrm{MHz}$ to transfer the 20 bit sample word, 4 bit control word, and 1 synchronization bit in $1 \mu \mathrm{s}$. We use $54 \mathrm{MHz}$ for controlling the SPI module and a $27 \mathrm{MHz}$ SPI clock for better timing margins which is well within the specifications of Category 6 cables.

\section{FPGA}

We use the Zybo Z7-20 development board [8] featuring a Xilinx XC7Z020-1CLG400C FPGA for the design and implementation of the real-time data transfer to four DAC channels. The PMOD ports of the Zybo board are used to transfer samples to DAC chips using up to four parallel SPI interfaces via a custom breakout board that connects the PMOD ports to the Ethernet cables. The proposed digital hardware is implemented with Verilog HDL and Vivado 2019.1.

\section{A. The Proposed Design}

The block diagram of the FPGA-based digital system implementing the control of the DACs is presented in Figure 3. The ARM processor of the Zynq-7000 SoC is the main controller of the FPGA architecture. It first receives the metadata via a TCP/IP based Ethernet connection from the PC at the initialization process and writes them to the $M D-B R A M \mathrm{~s}$ through Advanced eXtensible Interface (AXI) bus and interconnect. The MD-BRAM modules are implemented with onchip Block RAMs and will only store the metadata needed for the precise timing of each DAC channel's waveform samples. Afterwards, the ARM processor receives the DAC waveform samples from the same Ethernet connection and writes them to the DDR3. Each Ethernet packet contains a header to inform the ARM processor about the structure of the packet, allowing the processor to correctly extract the data and allocate the corresponding target addresses in the memory to each channel. DMA modules, which provide fast and continuous data transfers from the DDR3 to their corresponding DAC channel, are set by the ARM processor separately to read from their allocated memory segments in the DDR3. Each DMA module continuously reads the consecutive samples for their DAC channel and only pauses when the FIFO is full. The FIFO module serves as a buffer between DMA and SPI modules and maintains clock domain crossing. Furthermore, each FIFO is 24 bits wide and has a depth of 1024, where we use 20 bits to store the DAC samples. The DMA works at $125 \mathrm{MHz}$, making it faster than the Parsing/SPI modules and the DAC output activation which operate at $54 \mathrm{MHz}$ and $1 \mathrm{MHz}$, respectively. Therefore, the FIFO is never empty during the experiment, ensuring interruption-free data streaming to the DAC chips. The metadata provided by the $M D-B R A M \mathrm{~s}$ together with the waveform samples from the FIFOs is used by the Parsing module to control the SPI module.
In our current setup, the available $1 \mathrm{~GB}$ memory in the DDR3 is split into four parts to consecutively write the samples to four channels, while the memory allocated to each channel $i$ is split into $N_{i}$ parts to store the samples for $N_{i}$ waveforms or so-called Events. A visualization for $M$ channels is given in Figure 4. The first address of an $M D$-BRAM contains information about the total number of Events for the channel it belongs to. Each of the next address pairs are used to store the information about the number of samples (nSamples) in the waveform and the Periods of each sample for the corresponding event. The Periods parameter is simply given by the ratio of the maximum sampling frequency $(1 \mathrm{MHz})$ and the desired sampling frequency. Setting Periods to a value larger than 1 is useful for the user to consume less memory in the DDR3 when the generated signal is much slower than the $1 \mathrm{MHz}$ DAC refresh rate and allows for the user to record more waveforms in the DDR3.

A state machine is implemented in the Parsing module to control the SPI interface for each channel independently using the metadata. Initially, the Parsing module sends a read-enable signal to the FIFO to read the next 20 bit sample, prepends a 4 bit DAC control header to the sample data and transmits 24 bits of data to the SPI module together with a Sync 0 flag. The Sync 0 is a write-enable signal for the SPI module and a wait command for the DAC chip, thereby opening the window for writing a new sample to the chip. The SPI module transmits the data and control bits to the DAC chip in 24 cycles in serial. After the least significant bit of the sample is sent, the Parsing module sets the SYNC signal high, which closes the sampling window and triggers the DAC chip to change the analog output according to the written 20 bits of data. An example timing diagram is given in Figure 5. Meanwhile, an SPI clock $(S C L K)$ is generated at half the frequency $(27 \mathrm{MHz})$ of the FPGA clock (ACLK, $54 \mathrm{MHz})$. The initial Sync 0 output of the Parsing module starts with the rising edge of the external trigger signal, which provides synchronization across multiple channels, simultaneously processing the data for the DACs. Subsequently, the SYNC signal is periodically switched between 1 for updating the analog output of the DAC and 0 for loading the next sample, timed by the Parsing module's state machine, such that the resulting sampling frequency is $1 \mathrm{MHz}$. By utilizing a joint trigger and synchronous counters with the same $54 \mathrm{MHz}$ clock, the four channels output samples at the exact same time while maintaining the flexibility of dynamically changing waveforms and sampling frequencies independently from each other. This is also one key advantage offered by our system compared to off-the-shelf arbitrary waveform generators, which typically do not provide the freedom to switch to the next waveforms independently for each channel.

\section{B. Implementation Results}

The design is synthesized using Vivado 2019.1 and mapped to a Xilinx XC7Z020-1CLG400C FPGA comprising $53 \mathrm{k}$ Look-Up-Tables (LUT), $106 \mathrm{k}$ DFFs and 140 BRAMs and a Zynq SoC including dual Core ARM Cortex A9 processors. 
The digital hardware consumes $21 \%$ of the LUTs, $11 \%$ of the DFF and $13 \%$ of the BRAM resources of the FPGA. One of the ARM processors is used for PC-FPGA communication, setting the DMAs, and writing the data to the DDR3 and BRAMs.

The Ethernet based PC-FPGA communication, the data-flow in the DDR3, DMAs and FIFOs, the state machines of Parsing modules and the synchronous SPI outputs are verified in realtime using the Vivado tools Integrate Logic Analyzer (ILA) and Software Development Kit (SDK) debugger. The available 1 GB DDR3 allows storing $250 \mathrm{M}$ samples in 32 bits format, and, having a depth of 2048, each of the four BRAMs can store up to 1023 Events. The extension of the number of Events can be achieved by increasing the depth of the MD-BRAMs. Many channels on different FPGAs can function synchronously using the same reference clock source and a common trigger.

\section{HARDWARE}

\section{A. FPGA and breakout board}

The Zybo Z7-20 uses PMOD ports for the digital outputs which are connected to Maxim Integrated MAX9123 level translators on the breakout board to convert the single ended FPGA outputs to low voltage differential signals (LVDS). The breakout board also contains a power supply which provides $24 \mathrm{~V}$ to the DAC boards. The three SPI signals and the power line are connected to a RJ45 socket which allows the convenient use of Ethernet cables for data transmission and powering of the DAC board. The SPI clock is provided by a single digital output to all DAC boards, while the trigger and data outputs are distinct for each DAC board.

\section{B. DAC board}

The main component of the DAC board, shown in Figure 6, is the Analog Devices AD5791 chip which provides 20 bit digital to analog conversion at up to 1 MSPS sampling rate. The voltage reference for the DAC is an LTC6655LN which has low enough noise to reach the required 20 bits of precision as well as low drift in temperature and time. The reference is buffered and inverted to provide a positive and negative reference for the AD5791 by a ADA4004-4 operational amplifier (opamp). The gain of the opamp can be adjusted such that the following output ranges can be set: $0-5 \mathrm{~V}, 0-10 \mathrm{~V}$, $\pm 10 \mathrm{~V}, \pm 5 \mathrm{~V}$. The output of the AD5791 is buffered by a Texas Instruments OP189 low noise, zero drift opamp which can provide sufficient current to operate most devices while retaining 20 bit precision. The isolation of grounds for the DAC chip is achieved with the APD1031, which includes three isolated DC-DC converters and 7 isolated digital channels. The DC-DC converter receives $24 \mathrm{~V}$ from the back-end and converts it to $\pm 15 \mathrm{~V}$ and $5 \mathrm{~V}$ which are linearly regulated to $\pm 13.5 \mathrm{~V}$ for the DAC chip and the operational amplifiers and subsequently to $10 \mathrm{~V}$ for the voltage reference using ultralow noise linear regulators (LT3045-1, LT3045 and LT3093). The $5 \mathrm{~V}$ output is used to operate the digital isolator of the APD1031 as well as the digital side of the DAC chip. On the non-isolated side the, LVDS signals from the back-end are converted to single ended $3.3 \mathrm{~V}$ signals using a MAX9122 level translator. We have placed the components on a 6layer printed circuit board (PCB) which has dedicated analog, digital, and power layers sandwiched between ground layers to achieve immunity from electromagnetic radiation and crosstalk from digital signals. The size of the PCB is on the order of a credit card in order to connect to the analog inputs of the laboratory equipment using no or very short wires.

\section{CONCLUSION}

We designed an analog signal generator platform which will be able to control a quantum gas experiment with high precision at high speeds. The FPGA-based digital hardware is verified in real-time, and the DAC cards will be verified together with the digital system when they are manufactured in the coming months.

In the future, we plan to extend the system to have more analog output channels at lower sampling rates and high precision data acquisition. The back-end FPGA development will allow to change the front-end architecture to data acquisition by replacing the DAC with an analog to digital converter (ADC) or extending the amount of output channels per frontend board at the cost of reducing the sampling rate.

\section{ACKNOWLEDGMENT}

We acknowledge the Swiss National Science Foundation (Grants No. 182650 and No. NCCR-QSIT) and European Research Council advanced grant TransQ (Grant No. 742579) for funding.

\section{REFERENCES}

[1] I. Bloch, J. Dalibard, and S. Nascimbène, "Quantum simulations with ultracold quantum gases," Nature Physics, vol. 8, no. 4, pp. 267-276, Apr. 2012. [Online]. Available: https://www.nature.com/articles/nphys2259

[2] S. Krinner, T. Esslinger, and J.-P. Brantut, "Two-terminal transport measurements with cold atoms," Journal of Physics: Condensed Matter, vol. 29, no. 34, p. 343003, 2017. [Online]. Available: http://stacks.iop.org/0953-8984/29/i=34/a=343003

[3] Y. Wang, K. Wang, E. F. Fenton, Y.-W. Lin, K.-K. Ni, and J. D. Hood, "Reduction of laser intensity noise over $1 \mathrm{MHz}$ band for single atom trapping," Opt. Express, vol. 28, no. 21, pp. 31 209-31215, Oct 2020. [Online]. Available: http://www.opticsexpress.org/abstract.cfm?URI=oe28-21-31209

[4] A. Trenkwalder, M. Zaccanti, and N. Poli, "A flexible control system for atomic, molecular and optical physics experiments," arXiv:2106.02889 [cond-mat, physics:physics], Jun. 2021, arXiv: 2106.02889. [Online]. Available: http://arxiv.org/abs/2106.02889

[5] A. Sitaram, G. K. Campbell, and A. Restelli, "Programmable System on Chip for controlling an atomic physics experiment," Review of Scientific Instruments, vol. 92, no. 5, p. 055107, May 2021, arXiv: 2102.11230. [Online]. Available: http://arxiv.org/abs/2102.11230

[6] B. Malek, Z. Pagel, X. Wu, and H. Müller, "Embedded Control System for Mobile Atom Interferometers," Review of Scientific Instruments, vol. 90, no. 7, p. 073103, Jul. 2019, arXiv: 1812.01028. [Online]. Available: http://arxiv.org/abs/1812.01028

[7] W. Setiawan, "Fermi Gas Microscope," Ph.D. dissertation, Harvard University, Aug. 2012, accepted: 2012-08-03T22:47:20Z. [Online]. Available: https://dash.harvard.edu/handle/1/9366602

[8] "Zybo Z7 - Digilent Reference." [Online]. Available: https://reference.digilentinc.com/programmable-logic/zybo-z7/start

[9] T. Stöferle, "Exploring atomic quantum gases in optical lattices," Ph.D. dissertation, ETH Zürich, 2005. [Online]. Available: httpshttps://www.research-collection.ethz.ch/handle/20.500.11850/57916 